PROCEEDINGS OF THE

AMERICAN MATHEMATICAL SOCIETY

Volume 128, Number 4, Pages 1207-1213

S 0002-9939(99)05089-3

Article electronically published on December 10, 1999

\title{
CONTINUUM MANY FRÉCHET TYPES OF HEREDITARILY STRONGLY INFINITE-DIMENSIONAL CANTOR MANIFOLDS
}

\author{
VITALIJ A. CHATYRKO AND ELŻBIETA POL \\ (Communicated by Alan Dow)
}

\begin{abstract}
In this note we construct a family of continuum many hereditarily strongly infinite-dimensional Cantor manifolds such that for every two spaces from this family, no open subset of one is embeddable into the other.
\end{abstract}

\section{INTRODUCTION}

All our spaces will be metrizable separable. A space $X$ is strongly infinitedimensional (shortly, s.i.d.) if there exists an infinite sequence $\left(A_{1}, B_{1}\right),\left(A_{2}, B_{2}\right), \ldots$ of pairs of disjoint closed sets in $X$ such that if $L_{i}$ is a partition between $A_{i}$ and $B_{i}$ in $X$, then $\bigcap_{i=1}^{\infty} L_{i} \neq \emptyset$. We call $X$ hereditarily strongly infinite-dimensional (shortly, h.s.i.d.) if every subspace of $X$ is either 0-dimensional or strongly infinitedimensional. The first example of a h.s.i.d. compactum was given by Rubin [14] (a simpler construction is presented in 6], Problem 6.1.G). If $X$ is a non-trivial continuum and all closed sets which disconnect $X$ are infinite-dimensional we call $X$ an infinite-dimensional Cantor manifold (by a theorem of Tumarkin [16], every h.s.i.d. compact space contains a h.s.i.d. Cantor manifold).

In this note we show that in the class of h.s.i.d. Cantor manifolds there are continuum many pairwise incomparable Fréchet dimensional types; cf. [8]. The incomparability of $X$ and $Y$ means that neither $X$ embeds in $Y$ nor $Y$ embeds in $X$.

A collection of continuum many pairwise incomparable Cantor manifolds without any non-trivial finite-dimensional subcontinua was indicated in 13 . However, in Section 4 we shall describe an example, obtained jointly with Roman Pol, of a compactum without 1-dimensional subcontinua, still containing a 1-dimensional subset (we did not find any examples to this effect in the literature). It cannot be excluded that this phenomenon occurs among the Cantor manifolds considered in [13. Our present construction combines some ideas from [5] and [13], and it involves the method of "condensation of singularities" which we discuss in Section 2 This technique yields in fact a stronger version of incomparability, explained in Remark 3.3

Received by the editors January 13, 1998 and, in revised form, May 9, 1998.

2000 Mathematics Subject Classification. Primary 54F45.

Key words and phrases. Hereditarily strongly infinite-dimensional, Cantor manifolds, continuum, embedding, incomparable spaces. 
Our terminology follows [6]. By an embedding we shall mean a homeomorphic embedding, and by a continuum we should mean a non-trivial connected compactum. The symbol $|S|$ stands for the cardinality of $S$.

\section{REPLACING POINTS BY CONTINUA}

We shall apply a classical idea of "condensation of singularities". To this end we shall use the inverse system technique, following Anderson and Choquet [2]; cf. also Maćkowiak 12], Theorem 30. An alternative approach to the same effect can be based on Fedorčuk's method of "resolutions" (cf. [18]), as modified by the first author in 4] and [5. We shall need the following fact established by Aarts and van Emde Boas [1].

Lemma 2.1. Let $E$ and $K$ be continua and let $t$ be a point in $E$. There exists a continuum $S(E, K, t)$ and a continuous surjection $p: S(E, K, t) \longrightarrow E$ such that

(i) $p^{-1}(t)$ is a copy of $K$, boundary in $S(E, K, A)$, and the other fibers of $p$ are singletons,

(ii) if $L$ is a continuum in $S(E, K, t)$ such that $p(L)$ is non-trivial and it contains $t$, then $p^{-1}(t) \subset L$.

For the sake of completeness, let us outline the construction from [1] (cf. [5]). Embed $K$ in the hyperplane $I^{\infty} \times\{0\}$ of the product of the Hilbert cube and the interval $I$. Let $h:[0,+\infty) \longrightarrow I^{\infty} \times(0,1]$ be a homeomorphic embedding such that each neighbourhood of $K$ contains some ray $L_{n}=h([n,+\infty))$ and $\overline{L_{n}} \supset K\left(L_{0}\right.$ is a polygonal "spiral" approximating $K$ from above). Then define a continuous map $f: E \backslash\{t\} \longrightarrow L_{0}$ so that for each $1 / n$-neighbourhood $U_{n}$ of $t, f\left(U_{n} \backslash\{t\}\right) \subset L_{n}$. Finally, let $S(E, K, t)$ be the closure of the graph of $f$ in $E \times I^{\infty} \times I$, and let $p$ be the projection onto $E$.

2.2. The space $S(E, K, A)$. Let $E$ and $K$ be continua, and let $A \subset E$ be countable. Let $A=\left\{x_{1}, x_{2}, \ldots\right\}$, where $x_{i} \neq x_{j}$ for $i \neq j$. We shall define an inverse sequence $p_{i}: E_{i} \longrightarrow E_{i-1}$ with $E_{0}=E$ such that for $\pi_{i}=p_{i} \circ p_{i-1} \circ \ldots \circ p_{1}$ the fibers $\pi_{i}^{-1}\left(x_{j}\right)$ are copies of $K$, boundary in $E_{i}$, for $j \leq i$, the other fibers of $\pi_{i}$ are singletons, and for each continuum $L$ in $E_{i}$ with $x_{j} \in \pi_{i}(L)$ for some $j \leq i$, either $L \subset \pi_{i}^{-1}\left(x_{j}\right)$ or $L \supset \pi_{i}^{-1}\left(x_{j}\right)$. Having defined $E_{i}$ we take $t=\pi_{i}^{-1}\left(x_{i+1}\right)$, and let $E_{i+1}=S\left(E_{i}, K, t\right), p_{i+1}=p$, where $p: S\left(E_{i}, K, t\right) \longrightarrow E_{i}$ is the map from Lemma 2.1. Let $p: S(E, K, A) \longrightarrow E$ be the projection from the inverse limit $S(E, K, A)=\lim _{(}\left(E_{i}, p_{i}\right)$.

We shall keep this notation throughout the paper.

In a few subsequent lemmas we shall list the properties of $S(E, K, A)$ and the projection $p$ which are basic for our construction. Let us notice that an alternative construction described in [5] displays similar features.

Lemma 2.3. (i) Every fiber $p^{-1}(x)$, where $x \in A$, is a copy of $K$ and the other fibers are singletons. $K$.

(ii) If $A$ is dense in $E$, then every open subset of $S(E, K, A)$ contains a copy of

(iii) $p^{-1}(E \backslash A)$ is a dense subspace of $S(E, K, A)$ homeomorphic to $E \backslash A$.

This follows immediately from the construction.

Lemma 2.4. If $L$ is a continuum in $S(E, K, A$, ) and $p(L) \cap A \neq \emptyset$, then either $L$ embeds in $K$ or $K$ embeds in $L$. 
Proof. Let $L$ be a continuum in $S(E, K, A)$ with $x_{i} \in p(L)$ and let $q_{i}: S(E, K, A)$ $\longrightarrow E_{i}$ be the projection. Then $K_{i}=\pi_{i}^{-1}\left(x_{i}\right)$ is a copy of $K$ and either $q_{i}(L) \subset K_{i}$ or $K_{i} \subset q_{i}(L)$, by the construction. Since the fibers $\left(p_{i+k} \circ \ldots \circ p_{i}\right)^{-1}(s)$ are singletons for all $s \in K_{i}$ and $k=1,2, \ldots$, the map $q_{i}$ restricted to $q_{i}^{-1}\left(K_{i}\right)$ is injective and hence $q_{i}^{-1}\left(K_{i}\right)$ is a copy of $K$. Also, either $q_{i}^{-1} q_{i}(L)=L \subset q_{i}^{-1}\left(K_{i}\right)$ or $q_{i}^{-1}\left(K_{i}\right) \subset L$.

Lemma 2.5. If $E$ is an infinite-dimensional Cantor manifold, then so is $S(E, K, A)$

Proof. By Lemma 2.3(iii), every partition $F$ in $S(E, K, A)$ contains topologically $p(F) \backslash A$. Note that $p(F)$ is a partition in $E$. But $E$ is a Cantor manifold and $A$ is countable. Hence $p(F) \backslash A$ is infinite-dimensional. It follows that $F$ is infinitedimensional.

Lemma 2.6. If $K$ and $L$ are incomparable continua which do not embed in $E$ and $A$ is a countable dense subset of $E$, then no open non-empty subset of $S(E, L, A)$ embeds in $S(E, K, A)$.

Proof. Suppose that some non-empty open subset of $S(E, L, A)$ embeds in $S(E, K, A)$. Then by Lemma 2.3(ii) there exists an embedding $h: L \longrightarrow S(E, K, A)$. Since $L$ does not embed in $E, h(L) \cap p^{-1}(A) \neq \emptyset$. By Lemma 2.4, either $h(L)$ embeds in $K$ or vice versa, which provides a contradiction.

Let us recall that a space is hereditarily infinite-dimensional (h.i.d.) if it does not contain any subsets of positive finite dimension (first example of h.i.d. compactum was given by Walsh [17]). Evidently, every h.s.i.d. space is h.i.d. In the next lemma and also in the sequel we shall use the following simple observation: any countable union $X$ of h.i.d. (h.s.i.d.) compacta $X_{1}, X_{2}, \ldots$ is itself h.i.d. (h.s.i.d.). Indeed, if $A \subset X$ is finite dimensional (is not s.i.d.), then every $A \cap X_{i}$ is finite dimensional (is not s.i.d.); thus $A \cap X_{i}$ is 0 -dimensional for every $i \in N$ and by the sum theorem (cf. [6], Theorem 1.5.3) $A$ must be 0-dimensional.

Spaces which are not s.i.d. are called weakly infinite-dimensional (w.i.d.).

Lemma 2.7. If $E$ and $K$ are h.i.d. (respectively, h.s.i.d.), then $S(E, K, A)$ is h.i.d. (respectively, h.s.i.d.).

Proof. First note that $E_{i}$ is the union of finitely many compacta $\pi_{i}^{-1}\left(x_{j}\right), j=$ $1,2, \ldots, i$, each homeomorphic to $K$, and a set homeomorphic with an $F_{\sigma}$-subset of $E$. Hence if $E$ and $K$ are h.i.d. (h.s.i.d.), then so is $E_{i}$.

To begin with let us consider the case when $E$ and $K$ are h.i.d. Let $Y$ be a subset of $S(E, K, A)$ of dimension greater than 0 . Then there exists $i \in N$ such that the projection $q_{i}(Y)$ on $E_{i}$ has dimension greater than 0 . Since $E_{i}$ is h.i.d., $q_{i}(Y)$ must be infinite-dimensional. Thus the set $B=q_{i}(Y) \backslash C$, where $C=\bigcup_{j=i+1}^{\infty} \pi_{i}^{-1}\left(x_{j}\right)$ is countable, is infinite-dimensional and $q_{i} \mid q_{i}^{-1}(B)$ is injective, so $q_{i}^{-1}(B)$ is an infinite-dimensional subset of $Y$. Hence $Y$ is infinite-dimensional which ends the proof that $S(E, K, A)$ is h.i.d.

Suppose now that $E$ and $K$ are h.s.i.d. As was already proved, $S(E, K, A)$ is h.i.d. By Lemma 2.3 the space $S(E, K, A)$ is the union of countably many sets $p^{-1}\left(x_{i}\right), i=1,2, \ldots$, which are copies of $K$, and the subspace $p^{-1}(E \backslash A)$ homeomorphic to $E \backslash A$. Let $Y$ be a subset of $S(E, K, A)$ which is w.i.d. Then for every $i \in N$ the set $Y \cap p^{-1}\left(x_{i}\right)$ must be 0 -dimensional. Thus $D=\bigcup_{i=1}^{\infty}\left(Y \cap p^{-1}\left(x_{i}\right)\right)$ 
is 0-dimensional by the sum theorem and there exists a 0 -dimensional $G_{\delta}$-subset $G$ of $Y$ containing $D$; cf. [6], Theorem 1.5.11. The set $Y \backslash G$ is w.i.d. as an $F_{\sigma^{-}}$ subset of $Y$. But $Y \backslash G$ lies in a h.s.i.d. space $E$, hence it must be 0-dimensional. Therefore, $Y=(Y \backslash G) \cup(Y \cap G)$ is the union of two 0-dimensional sets, hence it is at most 1-dimensional. Since $S(E, K, A)$ is h.i.d., $\operatorname{dim} Y \leq 0$. And this completes the proof that $S(E, K, A)$ is h.s.i.d.

The last lemma will be used only in Section 4, Let us recall that $X$ is punctiform if $X$ contains no continuum; see [11, [6].

Lemma 2.8. If $E \backslash A$ is punctiform and $K$ is an infinite-dimensional continuum without 1-dimensional (respectively, w.i.d.) subcontinua, then $S(E, K, A)$ contains no 1-dimensional (respectively, w.i.d.) continua.

Proof. Let $L$ be a continuum in $S(E, K, A)$. Since $E \backslash A$ is punctiform, $L$ is not contained in $p^{-1}(E \backslash A)$. Hence $p(L) \cap A \neq \emptyset$ and by Lemma 2.4 either $L$ embeds in $K$ or vice versa. In both cases $L$ is infinite-dimensional (respectively, is s.i.d.).

\section{MAIN RESUlT}

We shall prove in this section our main

Theorem 3.1. There exists a family $\{Z(s): s \in \mathcal{S}\}$, where $|\mathcal{S}|=2^{\aleph_{0}}$, of hereditarily strongly infinite-dimensional Cantor manifolds such that for any distinct $s, s^{\prime} \in \mathcal{S}$, no non-empty open subset of $Z(s)$ embeds in $Z\left(s^{\prime}\right)$.

We shall start from the following auxiliary

Lemma 3.2. Let $A_{1}, A_{2}, \ldots$ be a sequence of continua, which cannot be separated by a point and such that $A_{j}$ does not embed in $A_{i}$ if $j>i$. Then there exists a family $\{X(s): s \in \mathcal{S}\},|\mathcal{S}|=2^{\aleph_{0}}$, such that $X(s)$ does not embed in $X\left(s^{\prime}\right)$ if $s \neq s^{\prime}$ and every $X(s)$ is a countable union of some $A_{i}$ compactified by a point.

Proof. For every sequence of natural numbers $s=\left\{n_{i}\right\}_{i=1}^{\infty}$, where $n_{1}<n_{2}<\ldots$, we shall define a continuum $X(s)$ which is a countable union of copies of some $A_{i}$ and a single point not in the union, in such a way that

(1) if sequences $s$ and $s^{\prime}$ are almost disjoint, then $X(s)$ does not embed in $X\left(s^{\prime}\right)$.

First, choose inductively natural numbers $\kappa(1), \kappa(2), \ldots$ in such a way that

(2) $\quad \kappa(j)>\sum_{i=1}^{j-1} \kappa(i)$ for every $j \in N$.

In every $A_{i}$ choose two different points $a_{1}^{i}, a_{2}^{i}$. If $s=\left\{n_{i}\right\}_{i=1}^{\infty}$ is an increasing sequence of natural numbers, then let $Y_{1}^{s}, Y_{2}^{s}, \ldots$ be a sequence of spaces such that $Y_{p}^{s}=A_{n_{1}}$ for $1 \leq p \leq \kappa\left(n_{1}\right)$ and $Y_{p}^{s}=A_{n_{i}}$ for $\kappa\left(n_{1}\right)+\ldots+\kappa\left(n_{i-1}\right)<$ $p \leq \kappa\left(n_{1}\right)+\ldots+\kappa\left(n_{i-1}\right)+\kappa\left(n_{i}\right)$ (i.e., the first $\kappa\left(n_{1}\right)$ terms in this sequence are homeomorphic to $A_{n_{1}}$, the next $\kappa\left(n_{2}\right)$ terms are homeomorphic to $A_{n_{2}}$, and so on). For every $i \in N$, if $Y_{i}^{s}=A_{j}$, then let $x_{1}^{i}=a_{1}^{j}$ and $x_{2}^{i}=a_{2}^{j}$. Consider the equivalence relation $E$ on the discrete sum $Y(s)=\bigoplus_{i=1}^{\infty} Y_{i}^{s}$ defined by $x E y$ iff $x=y$ or $x=x_{2}^{i}$ and $y=x_{1}^{i+1}$ for some $i \in N$. Let $Z(s)=Y(s) / E$ be the quotient space, let $q: Y \longrightarrow Z(s)$ be the quotient mapping and finally let $X(s)$ be the one-point compactification of $Z(s)$. The space $X(s)$ is a continuum. Notice that $X(s)$ is a one-point compactification of a chain $X_{1}^{s}, X_{2}^{s}, \ldots$ of continua, where $X_{i}^{s}=q\left(Y_{i}^{s}\right)$ is homeomorphic to $Y_{i}^{s}$. The term chain refers here and in the sequel 
to the union of a sequence (finite or infinite) of spaces such that only the spaces with subsequent indices meet and the intersection is a singleton.

Let us prove (1). Let $s=\left\{n_{i}\right\}_{i=1}^{\infty}$ and $s^{\prime}=\left\{k_{j}\right\}_{j=1}^{\infty}$ be two increasing sequences of natural numbers which are almost disjoint, i.e. there exists $n_{0}$ such that for every $n_{i}, k_{j}>n_{0}$ we have $n_{i} \neq k_{j}$.

Suppose that there exists an embedding $f: X(s) \longrightarrow X\left(s^{\prime}\right)$. Take $i_{0}$ such that $n_{i_{0}}>n_{0}$. Then $X_{\kappa\left(n_{1}\right)+\kappa\left(n_{2}\right)+\ldots+\kappa\left(n_{i_{0}}\right)}=A_{n_{i_{0}}}$ and there exist $j, j_{0} \in N$ such that

$$
f\left(X_{\kappa\left(n_{1}\right)+\ldots+\kappa\left(n_{i_{0}}\right)}\right) \subset X_{j}^{s^{\prime}}=A_{k_{j_{0}}},
$$

where $\kappa\left(k_{1}\right)+\ldots+\kappa\left(k_{j_{0}-1}\right)+1 \leq j \leq \kappa\left(k_{1}\right)+\ldots+\kappa\left(k_{j_{0}}\right)$. Let $i_{1}$ be the biggest natural number such that $n_{i_{1}}<k_{j_{0}+1}$ (such a number exists and $i_{1} \geq i_{0}$ ). Then $n_{i_{1}+1}>k_{j_{0}+1}$, which means that $X_{\kappa\left(n_{1}\right)+\ldots+\kappa\left(n_{i_{1}}\right)+1}=A_{n_{i_{1}+1}}$ cannot be embedded in any of $X_{m}^{s^{\prime}}$ for $m \leq \kappa\left(k_{1}\right)+\ldots+\kappa\left(k_{j_{0}+1}\right)$. This implies that the chain $X_{\kappa\left(n_{1}\right)+\ldots+\kappa\left(n_{i_{0}}\right)+1}^{s} \cup X_{\kappa\left(n_{1}\right)+\ldots+\kappa\left(n_{i_{0}}\right)+2}^{s} \cup \ldots \cup X_{\kappa\left(n_{1}\right)+\ldots+\kappa\left(n_{i_{1}}\right)}^{s}$ consisting of $\kappa\left(n_{i_{0}+1}\right)+\kappa\left(n_{i_{0}+2}\right)+\ldots+\kappa\left(n_{i_{1}}\right)$ continua from the family $\left\{A_{i}\right\}_{i=1}^{\infty}$ must be mapped into a chain of at least $\kappa\left(k_{j_{0}+1}\right)$ continua from this family in such a way that its image under $f$ intersects the first and the last element of this chain in more than one point. This is impossible by virtue of (1) and the fact that no $A_{i}$ can be separated by a point. The obtained contradiction proves (1).

Let $\mathcal{S}$ be an almost disjoint family of cardinality continuum consisting of increasing sequences of natural numbers (see [7, Example 3.6.18, for a simple construction of such a family). Then $\{X(s): s \in \mathcal{S}\}$ is a family of cardinality continuum consisting of pairwise incomparable continua.

Now we can pass to the

Proof of Theorem 3.1. To begin with we will show that

(3) for every h.s.i.d. continuum $X$ there exists a h.s.i.d. Cantor manifold $Y$ which does not embed in $X$.

Let $X$ be a h.s.i.d. continuum. We shall use the fact (cf. [13], Lemma 6.3) that for the compactum $X$ there exists a compactum $Z$ which is a countable union of topological copies of $X$ and does not embed in $X$. Of course, $Z$ is h.s.i.d. Take any h.s.i.d. Cantor manifold $H$ (see the Introduction), any Cantor set $C \subset H$ and any continuous surjection $f: C \longrightarrow Z$. Let $Y=H \cup_{f} Z$ be the adjunction space of $H$ and $Z$ under the map $f$, i.e. the quotient space $H / E$, where $E$ is an equivalence relation defined by $x E y$ iff $x=y$ or $x, y \in C$ and $f(x)=f(y)$. Then $Y$ is a h.s.i.d. Cantor manifold (cf. [13, Lemma 3.1) which does not embed in $X$.

(4) There exists a sequence $M_{0}, M_{1}, M_{2}, \ldots$ of h.s.i.d. Cantor manifolds such that if $j>i$, then $M_{j}$ does not embed in $M_{i}$.

This follows from (3) by induction. Let $M_{0}$ be any h.s.i.d. Cantor manifold. If $M_{0}, M_{1}, \ldots, M_{i}$ are already constructed, then we apply (3) to the bouquet $B$ of $M_{0}, M_{1}, \ldots, M_{i}$, i.e. $B=\bigcup_{j=0}^{i} M_{j}$, where each $M_{i}$ and $M_{j}$ with distinct $i, j$ meet at a singleton $\{p\}$.

By Lemma 3.2 there exists a family $\{X(s): s \in \mathcal{S}\}$, where $|\mathcal{S}|=2^{\aleph_{0}}$, of pairwise incomparable continua such that every $X(s)$ is a countable union of some $M_{i}$, for $i \geq 1$, and a point. Obviously, every $X(s)$ is h.s.i.d. and does not embed in $M_{0}$.

For every $s \in S$ put $Z(s)=S\left(M_{0}, X(s), A\right)$, where $A$ is any countable dense subset of $M_{0}$. Then every $Z(s)$ is a h.s.i.d. Cantor manifold by Lemmas 2.5$]$ and 2.7 
and if $s \neq s^{\prime}$, then no non-empty open subset of $Z(s)$ embeds in $Z\left(s^{\prime}\right)$ by Lemma 2.6.

Remark 3.3. K. Borsuk [3] called two spaces $X$ and $Y$ locally $r$-incomparable if no non-empty open subset of one can be embedded into the other. Therefore Theorem 3.1 can be stated in the following way.

There exist continuum many pairwise locally r-incomparable h.s.i.d. Cantor manifolds.

For some related results concerning local $r$-incomparability in various classes of spaces the reader is refered to $[5]$.

\section{A compactum without 1-Dimensional continua WHICH CONTAINS A CONNECTED 1-DIMENSIONAL SET}

Henderson 9] constructed in 1967 continua without 1-dimensional subcontinua. Due to the complexity of the construction it is unclear whether or not the Henderson's compacta, or their subsequent modifications, contain 1-dimensional subsets. In this section we provide an example, obtained jointly with Roman Pol, of a Henderson's compactum which contains a 1-dimensional connected subset and hence it is not h.i.d.

Example 4.1. There exists a continuum $Z$ all of whose non-trivial subcontinua are s.i.d., but which contains a 1-dimensional connected subset.

Proof. Let $E$ be a continuum containing a countable set $A$ such that $E \backslash A$ is punctiform and it contains a 1-dimensional connected subspace $G$. Take any continuum $K$ all of whose non-trivial subcontinua are s.i.d. (see [10]; cf. 6], Problem 6.1.G). Let $Z=S(E, K, A)$ and $p: Z \longrightarrow E$ be the continuum and the projection described in 2.2. Then $Z$ has the required properties. Indeed, by Lemma 2.3(i), $p$ restricted to $p^{-1}(E \backslash A)$ is a homeomorphism onto $E \backslash A$, so $G$ embeds in $Z$. By Lemma 2.7 $Z$ contains no w.i.d. subcontinua.

It remains to indicate a continuum $E$ with the required properties. Let $G$ be the Erdös space consisting of the points in the Hilbert space with all coordinates irrational, embedded in the Cantor fan $F(C)$ and augmented by a point as in [14; cf. [6], Examples 1.2.15 and 1.4.6 and Exercise 1.4.B. Here, $F(C)$ is the union $\bigcup_{c \in C} L_{c}$ of the segments $L_{c}$ in the square $I \times I$ joining the vertex $(1 / 2,1)$ with the points $c$ from the Cantor set $C$ in the unit interval $I \times\{0\} \subset R^{2}$. The space $G$ is a punctiform and 1-dimensional $G_{\delta}$-subset of $F(C)$. We shall show that there exists a continuum $E$ containing a countable set $A$ such that $E \backslash A$ is punctiform and contains topologically $G$.

Indeed, let $Q$ be the set of rationals in $I$, and let diam and dist stand for the diameter and distance with respect to the euclidean metric in $F(C)$. Since $G$ is $G_{\delta}$ we have

$$
F=(I \times Q) \cap(F(C) \backslash G)=\bigcup_{i=1}^{\infty} F_{i},
$$

where $F_{i}$ are compact and zero-dimensional. For every $i \in N$, the countable cover $\left\{U_{i j}\right\}_{j=1}^{\infty}$ of $F_{i} \backslash\left(F_{1} \cup \ldots \cup F_{i-1}\right)$, where $U_{i j}=\left\{x \in F_{i}: \operatorname{dist}\left(x, F_{1} \cup \ldots \cup F_{i-1}\right)>1 / i\right\}$, has a shrinking $\left\{F_{i j}\right\}_{j=1}^{\infty}$ consisting of disjoint compact sets (cf. [11], $\S 26$.II or [6], Proposition 3.2.2). Splitting each set $F_{i j}$ into finitely many relatively closed-andopen sets of diameter $\leq \frac{1}{i+j}$, we get a disjoint cover $A_{1}, A_{2}, \ldots$ of $F$ by compact 
sets with $\operatorname{diam} A_{i} \rightarrow 0$. Let $\mathcal{D}=\left\{A_{i}: i=1,2, \ldots\right\} \cup\{x: x \notin F\}$ be an upper semi-continuous decomposition of $F(C)$, let $E=F(C) / \mathcal{D}$ be the quotient space, let $q: F(C) \longrightarrow E$ be the quotient map and $A=q(F)$. Then $q(G) \subset E \backslash A$ is homeomorphic to $G$. It remains to show that every continuum $L$ in $E$ intersects $A$, i.e. $E \backslash A$ is punctiform. If $L \cap A=\emptyset$, then $q^{-1}(L)$ is a continuum in $F(C) \backslash F$ homeomorphic to $L$. Thus $q^{-1}(L)$ contains a subinterval $J$ of some ray $L_{c}$. But $G \cap L_{c}$ is a finite set (in fact, one or two point set), so $(I \times Q) \cap J$ must intersect $F$. The contradiction completes the proof.

\section{REFERENCES}

[1] J.M.Aarts and P. van Emde Boas, Continua as remainders in compact extensions, Nieuw Arch. Wisk. 15 (1967), 34 - 37. MR 35:4885

[2] R.D.Anderson and G.Choquet, A plane continuum no two of whose nondegenerate subcontinua are homeomorphic: an application of inverse limite, Proc. Amer. Math. Soc. 10 (1959), 347 - 353. MR 21:3819

[3] K.Borsuk, Theory of Retracts, PWN, Warszawa 1969. MR 35:7306

[4] V.A.Chatyrko, On hereditarily indecomposable non-metrizable continua, Mat. Zametki 46 (1989), 122 - 125 (in Russian). MR 91a:54044

[5] V.A.Chatyrko, On locally $r$-incomparable families of infinite-dimensional Cantor manifolds, Comment. Math. Univ. Carolinae 40, 1 (1999), 165 - 173.

[6] R.Engelking, Theory of dimensions, finite and infinite, Heldermann Verlag, Lemgo, 1995. MR 97j:54033

[7] R.Engelking, General Topology, Helderman Verlag, Berlin 1989. MR 91c:54001

[8] M.Fréchet, Les dimensions d'un ensemble abstrait, Mathematishe Annalen 68 (1910), 145 168.

[9] D.W.Henderson, An infinite-dimensional compactum with no positive-dimensional compact subsets - a simpler construction, Amer. Journ. Math. 89 (1967), 105 - 121. MR 35:967

[10] D.W.Henderson, Each strongly infinite-dimensional compactum contains a hereditarily infinite-dimensional compact subset, Amer. Journ. Math. 89 (1967), 122 - 123. MR 35:968

[11] K.Kuratowski, Topology, vols. I, II, PWN, Warszawa 1966, 1968. MR 41:4467

[12] T.Maćkowiak, The condensation of singularities in arc-like continua, Houston Journ. Math. 11, 1985, 535 - 558. MR 87m:54099

[13] E.Pol, On infinite-dimensional Cantor manifolds, Topology and Appl. 71 (1996), 265 - 276. MR 97d:54059

[14] J.H.Roberts, The rational points in Hilbert space, Duke Math. Journ. 23 (1956), 489 - 492. MR 18:55b

[15] L.R.Rubin, Hereditarily strongly infinite dimensional spaces, Michigan Math. Journ. 27 (1980), 65 - 73 MR 80m:54050

[16] L.A.Tumarkin, On Cantorian manifolds of an infinite number of dimensions, DAN SSSR 115 (1957), 244 - 246 (in Russian) MR 19:971h

[17] J.J.Walsh, An infinite dimensional compactum containing no $n$-dimensional $(n \geq 1)$ subsets, Topology 18 (1979), 91 - 95. MR 80e:54050

[18] S.Watson, The construction of topological spaces: planks and resolutions, in: Recent Progress in General Topology, ed. M.Hušek and J.van Mill, Elsevier 1992, pp. 675 - 757. number of dimension, DAN SSSR 115 (1957), 244-246 (in Russian). MR 93:15

NiIsi Ran, Pattern Recognition and Expert Systems Division, Moscow, Russia Current address: Department of Mathematics, Linkoping University, 58183 Linkoping, Sweden E-mail address: vitja@mai.liu.se

Institute of Mathematics, University of WarsaW, Banacha 2, 02-097 Warszawa, Poland E-mail address: pol@mimuw.edu.pl 